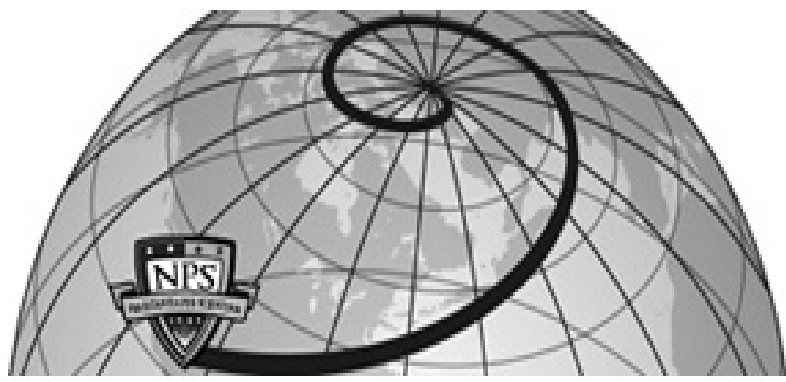

Calhoun: The NPS Institutional Archive DSpace Repository

\title{
Electron beam effects in UV FEL
}

Wong, R.K.; Blau, J.; Colson, W.B.

Nuclear Instruments and Methods in Physics Research A, Volume 375, (1996), pp. 356-357

https://hdl.handle.net/10945/44070

This publication is a work of the U.S. Government as defined in Title 17, United States Code, Section 101. Copyright protection is not available for this work in the United States.

Downloaded from NPS Archive: Calhoun

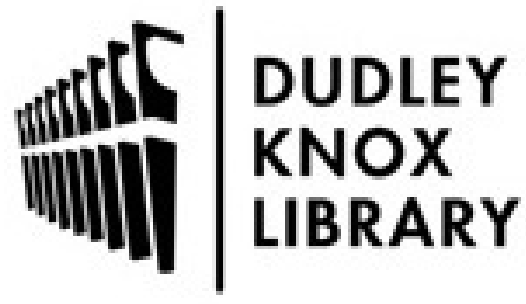

http://www.nps.edu/library
Calhoun is the Naval Postgraduate School's public access digital repository for research materials and institutional publications created by the NPS community. Calhoun is named for Professor of Mathematics Guy K. Calhoun, NPS's first appointed -- and published -- scholarly author.

Dudley Knox Library / Naval Postgraduate School 411 Dyer Road / 1 University Circle Monterey, California USA 93943 


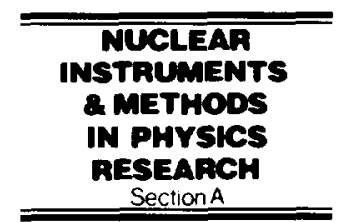

\title{
Electron beam effects in UV FEL
}

\author{
R.K. Wong, J. Blau, W.B. Colson* \\ Physics Department, Naval Postgraduate School, Monterey, CA 93943, USA
}

The Laser Processing Consortium (LPC) proposes to build a UV FEL at the Continuous Electron Beam Accelerator Facility (CEBAF) (see these proceedings, G. Neil et al.). The initial experiment is to provide $1 \mathrm{~kW}$ of average power. The electron beam will have an energy of $\gamma=$ $200 \mathrm{MeV}$, a peak current of $I=270 \mathrm{~A}$, a pulse length of $0.2 \mathrm{ps}$, and an emittance of $8-14 \pi \mathrm{mm} \mathrm{mrad}$. The output wavelength $\lambda=204 \mathrm{~nm}$, using a linear undulator with a period of $3.3 \mathrm{~cm}$ and an undulator parameter of $K=0.95$. These operating parameters lead to an electron beam radius of the order of the optical mode size. The performance of this FEL is sensitive to the transverse motion of electrons in the optical mode caused by betatron and external focusing. Additionally longitudinal effects may be important as the electron pulse length is on the order of a few slippage distances.

A 4D FEL simulation uses FEL theory based on a self-consistent solution to the coupled Maxwell-Lorentz equations describing the evolution of the optical pulse in a FEL oscillator over many passes [1]. A diffraction operator takes into the transverse optical mode effects in both the $x$ and $y$ directions as it is driven by an electron beam experiencing focusing in the transverse direction. The electron beam and optical pulse has finite dimensions in the longitudinal direction. The interaction is followed as the light propagates in a resonator configuration that accounts for losses and output coupling. Dimensionless parameters [1] are used to simplify the description of the physical interaction.

In Fig. 1, the results of a 4D FEL simulation shows the optical pulse shape $|a(z, n)|$ after 100 passes in steady state saturation.

The transverse optical wavefront $|a(x, y)|$ at the center of the optical pulse at exiting the undulator is shown in the far right diagram. The transverse density of the electron bearn driving the wavefront is superimposed in gray for comparison. The center diagram shows the optical power spectrum $P(\nu, n)$ evolution, with the final linewidth of $\Delta \lambda / \lambda=-\Delta \nu /(2 \pi N)=0.5 \%$. The middle diagams show the evolution of the optical pulse from pass $n=1$ to 100 . The bottom left diagram is the longitudinal profile of the

* Corresponding author. Tel. +1 4086562896 , fax +1408656 2834, e-mail colson@physics.nps.navy.mil. current density $j(z+\tau)$, where the dimensionless time $\tau$ goes from 0 to 1 as the electron travels from the beginning to the end of the undulator. The Rayleigh range scaled to the undulator length is $z_{0}=0.25$. The output-coupling of the mirrors is described by $Q=3$ with a mirror edge loss of $e=0.01$. The electrons have a betatron frequency of $\omega_{\beta}=1$ indicating one-sixth of a full period through the $N=72$ period undulator. The normalized emittance for this simulation is $\varepsilon_{N}=11 \pi \mathrm{mm}$-mrad. The electron beam energy quality leads to a Gaussian spread in electron phase velocity of $\sigma_{G}=1.3$ and the effect of the emittance of the angular distribution leads to an exponential width of $\sigma_{\theta}=$ 2.2. The electron beam radius is $\sigma_{e}=0.4$ in units of the optical mode waist defined by $\sqrt{L \lambda / \pi}$. The off-axis electrons in the transverse $y$-direction cause a spread in electron phase velocity of $\sigma_{v}=0.4$. The weak-field, singlemode gain spectrum is shown for comparison in the bottom middle diagram with a peak gain of $G=0.135 j F=$ 1, where $F$ is the filling factor accounting for the overlap of the electron beam with the optical mode. The dimensionless optical power plot in the lower right is well into saturation corresponding to an output power of approximately $1 \mathrm{~kW}$. The output power increases by $25 \%$ as the emittance is improved from 11 to $8 \pi \mathrm{mm}$-mrad. Increasing the Rayleigh range from $z_{0}=0.25$ to $z_{0}=0.75$ leads to a $20 \%$ decrease in output power. Large desynchronism is

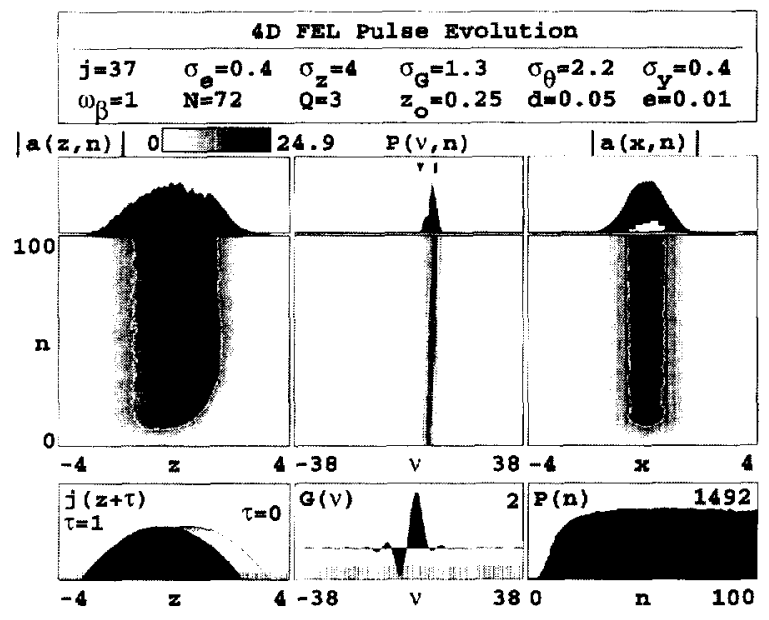

Fig. 1. 4D FEL showing the optical pulse after 100 passes. 
necessary for the pulse to counter lethargy in the steady state. In the longitudinal direction, the electron beam pulse length is $\sigma_{z}=4$, as measured in units of the slippage distance $N \lambda$. High output coupling causes lethargy to become important and the FEL operates at a high value of desynchronism, $d=0.05$ in units of the slippage distance. For higher $Q$ and lower emittance, the optical mode can result in higher operating powers, trapped particle instability and optical sidebands, broadening the optical spectrum. The effect of the output power on changing the $Q$ is small in the range of $Q=3-8$. Lowering the intracavity power

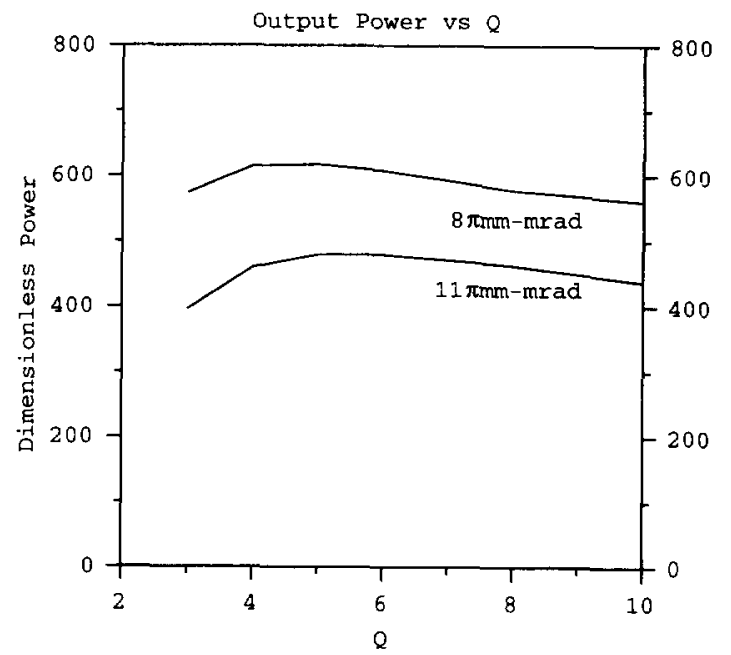

Fig. 2. Trend for increasing $\sigma_{i}$. by increasing the output coupling suggests that a low $Q$ is desirable to reduce power density at the mirrors.

The electron beam radius can be changed with constant emittance to optimize the interaction. The electron beam is chosen with betatron focusing in the $y$ direction and external focusing in $x$ direction. With constant emittance, increasing the electron beam size decreases the angular spread. The gain as a function of the electron beam radius peaks at $\sigma_{e}=0.3-04$ at the Rayleigh range of $z_{0}=0.25$ 0.5 and $\varepsilon=11 \pi \mathrm{mm}$-mrad. A smaller radius beam with higher angular spread leads to a broad spread in phase velocities, decreasing the gain. For a larger radius beam, poor overlap between the electron beam and optical mode reduces the gain. As the emittance increases, the peak gain occurs at higher electron beam radii.

\section{Acknowledgements}

The authors are grateful for the support of this work by the Naval Postgraduate School and SURA/CEBAF.

\section{References}

[1] W.B. Colson, Free Electron Laser Handbook, eds. W.B. Colson, C. Pellegrini and A. Renieri (North-Holland, Amsterdam, 1990) Chapt. 5. 